\section{Selbstausbeutung ist für junge Ärzte nicht selbstverständlich}

\author{
Angehende Mediziner liebäugeln mit dem Dasein als Angestellte in der \\ Gemeinschaftspraxis. Das ist nur ein Ergebnis einer Umfrage des Hartmann- \\ bundes.
}

$\mathrm{M}$ ehr Ärzte in der Versorgung - das geht nur, wenn der Arztberuf der Zukunft die Vereinbarkeit von Familie und Beruf garantiert. Auf diesen Nenner lassen sich die Ergebnisse der jüngsten Umfrage des Hartmannbundes unter seinen 20.000 studierenden Mitgliedern $65 \%$ davon sind Frauen - bringen. Die Präferenzen sind klar: $83 \%$ der Befragten knapp 4.400 angehenden Mediziner planen eine Familie oder bringen schon heute ein Baby mit in den Hörsaal. Da ist es folgerichtig, dass der Wunsch nach flexiblen Arbeitszeitmodellen und planbaren Arbeitszeiten eine wichtige Rolle spielt. Ein Wink mit dem Zaunpfahl in Richtung der Kliniken, die bei den Studierenden nach wie vor den Ruf haben, mit ausufernden Arbeitszeiten und hierarchischen Strukturen einer ausgeglichenen work life balance im Wege zu stehen. „Die Bereitschaft zur Selbstausbeutung bringt diese Generation nicht mehr wie selbstverständlich mit", so HartmannbundChef Dr. Klaus Reinhardt.

\section{Junge Mediziner bevorzugen Gemeinschaftspraxen und $\mathbf{M V Z}$}

„Die Ergebnisse der Umfrage sind ein deutlicher Weckruf an Politik und Klinikträger, angehenden Ärztinnen und Ärzten endlich attraktive berufliche Perspektiven zu bieten“, sagte Kristian Otte, Vorsitzender des Ausschusses der Medizinstudierenden im Hartmannbund. Als Angestellte in den ambulanten Sektor auszuweichen, ist für eine zunehmende Zahl junger Mediziner eine Alternative. Gemeinschaftspraxen und Medizinische Versorgungszentren (MVZ) liegen im Trend. Immerhin $40 \%$ halten die Arbeit, einschließlich der Teamarbeit, dort für befriedigender als in der Klinik sowie die Arbeitszeiten für flexibler. Jedenfalls dann, wenn sie nach dem Examen überhaupt in die Arbeit mit Patienten wechseln. Fast die Hälfte der Befragten kann sich vorstellen, außerhalb der kurativen Medizin tätig zu werden. Würde dies
Wirklichkeit, würde der Versorgungsnotstand den ländlichen Raum zuerst treffen. Nur 9\% der Befragten wollen eine Praxis auf dem Land übernehmen. Allerdings geben drei Viertel an, sich vorstellen zu können, wenigstens für ein paar Jahre, also befristet auf dem Land zu arbeiten. Die Hälfte würde dafür sogar bis zu 50 Kilometer Anfahrt zur Praxis in Kauf nehmen.

\section{Nachfolgersuche für Einzelpraxen wird schwer werden}

Nur 27\% planen heute schon die Niederlassung. Nur $10 \%$ sehen sich in einer Einzelpraxis. Heute besteht der niedergelassene Bereich noch zu rund $60 \%$ aus Einzelpraxen. Für diejenigen, die die Gemeinschafts- der Einzelpraxis vorziehen, sind das unternehmerische Risiko, die Bürokratie und wiederum die Familie dafür ausschlaggebend. „Das sind die $\mathrm{Zu}$ kunftsbaustellen der ärztlichen Versorgung“, so Otte. Ärzte, die diese nicht wahrnähmen, dürften sich nicht wundern, wenn sie keine Praxisnachfolger fänden, sagte Reinhardt.

\section{Angebote für Ärzte plus Lebenspartner von Interesse}

Ein Anreiz für angehende Mediziner: Kommunen müssten auf der Suche nach Ärzten auch den Partnern der Interessenten Arbeitsplätze anbieten und die Kinderbetreuung organisieren, sagte Reinhardt. Ähnliche Ergebnisse hatte bereits eine Umfrage der KBV und der Universität Trier im Jahr 2010 unter mehr als 12.000 angehenden Medizinern zu Tage gefördert. Deutlich mehr als $90 \%$ wünschten sich damals, Beruf und eine Familie mit Kindern gut unter einen Hut bringen zu können.

Teilzeitarbeit streben nicht ausschließlich nur Frauen an. Immerhin ein Drittel der befragten männlichen Studierenden zog noch vor zwei Jahren eine Arbeitszeit unterhalb der $100 \%$ vor.

Anno Fricke

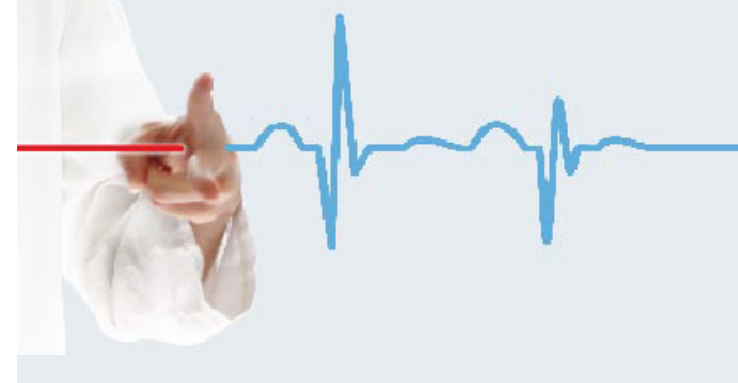

Wird nur gewerbsmäßige Sterbehilfe verboten?

Das Bundesjustizministerium hat einen Referentenentwurf vorgelegt, nach dem „gewerbsmäßige" Suizidbeihilfe mit bis zu drei Jahren Haft oder Geldstrafe belangt werden kann. Dazu soll § 217 Strafgesetzbuch entsprechend geändert werden. Damit zielt der Entwurf auf Sterbehilfeorganisationen, die sich durch "wiederholte Tatbegehung" der Suizidbeihilfe eine „fortlaufende Einnahmequelle" verschaffen. Nicht strafwürdig wäre dagegen etwa die Hilfe bei der Selbsttötung in der Familie oder die Suizidbeihilfe durch Ärzte. Bayern fordert dagegen, jede Form organisierter Sterbehilfe zu bestrafen. Die bayerische Justizministerin Beate Merk hat ein umfassendes Verbot gefordert, weil entsprechende Organisationen leicht ihr Ziel, Gewinne zu erzielen, verschleiern könnten.

\section{Patienten fühlen sich selten ernst genommen}

Krankenhäuser können ihren Umgang mit Patientenkritik noch deutlich verbessern. Etwa jeder fünfte Patient wendet sich während eines Krankenhausaufenthaltes an seinen Arzt, weil er mit der Behandlung unzufrieden ist. Das geht aus einer Forsa-Umfrage hervor, die im Auftrag der Schön Klinik aus Prien am Chiemsee vorgenommen wurde. Demnach fühlen sich aber nur 38\% der Patienten von ihrem Krankenhaus mit ihrem Anliegen ernst genommen. Männer äußerten ihre Kritik offenbar häufiger (21\%) als Frauen (15\%). Für die Umfrage wurden 500 Patienten zwischen 40 und 70 Jahren befragt.

$e b$ 